\title{
Long-term survival in asymptomatic patients with severe degenerative mitral regurgitation: A propensity score-based comparison between an early surgical strategy and a conservative treatment approach
}

\author{
Patrick Montant, MD, ${ }^{\mathrm{a}, *}$ Fabien Chenot, MD, ${ }^{\mathrm{a}, *}$ Annie Robert, PhD, ${ }^{\mathrm{b}}$ David Vancraeynest, MD, ${ }^{\mathrm{a}}$ \\ Agnès Pasquet, $\mathrm{MD}, \mathrm{PhD},{ }^{\mathrm{a}}$ Bernard Gerber, $\mathrm{MD}, \mathrm{PhD},{ }^{\mathrm{a}}$ Philippe Noirhomme, $\mathrm{MD},{ }^{\mathrm{a}}$ \\ Gébrine El Khoury, $\mathrm{MD},{ }^{\mathrm{a}}$ and Jean-Louis Vanoverschelde, $\mathrm{MD}, \mathrm{PhD}^{\mathrm{a}}$
}

\begin{abstract}
Aims: The management of asymptomatic severe mitral regurgitation remains controversial. The aim of the study was to assess the long-term survival, incidence of cardiac complications, factors that predict outcome, and effect of mitral surgery on the long-term prognosis of patients with asymptomatic severe mitral regurgitation amenable to valve repair.

Methods: One hundred ninety-two asymptomatic patients (mean age, $63 \pm 13$ years) with severe degenerative mitral regurgitation diagnosed by 2-dimensional echocardiography between 1990 and 2001 were prospectively followed for a median of 8.5 years.

Results: Overall, cardiovascular, and event-free survival was evaluated in 2 groups of patients: a "conservative approach" group $(n=67)$ and an "early surgery" group $(n=125)$. Outcomes were also analyzed among patients with atrial fibrillation, pulmonary hypertension, or both, as well as in patients free of any mitral regurgitation complications. In the whole population, 10-year overall survival was significantly lower with the conservative approach than early surgery $(50 \% \pm 7 \%$ vs $86 \% \pm 4 \%$, log-rank $<0.0001)$. Similar results were obtained in the subgroups with atrial fibrillation and/or pulmonary hypertension. The 10-year propensity-matched score-adjusted hazards ratio for overall mortality, cardiac mortality, and cardiovascular events for the conservative treatment were 5.21, 4.83, and 4.40, respectively.
\end{abstract}

Conclusion: Our results show that the outcome of asymptomatic patients with severe degenerative mitral regurgitation is better with an early surgical approach rather than a more conservative treatment strategy. ( $\mathrm{J}$ Thorac Cardiovasc Surg 2009;138:1339-48)

Earn CME credits at

http://cme.ctsnetjournals.org

Degenerative mitral regurgitation (MR) is the second most frequently encountered valvular disease in Western countries and is often discovered fortuitously in still asymptomatic patients. ${ }^{1,2}$ Although surgery is the only proven efficient therapeutic option for patients with severe MR, its

\footnotetext{
From the Division of Cardiology, Cliniques Universitaires, St-Luc, ${ }^{a}$ and Epidemiology and Biostatistics Unit, ${ }^{\text {b }}$ Université Catholique de Louvain, Brussels, Belgium.

Supported by the Fonds National de la Recherche Scientifique of the Belgian Government (FNRS). Dr Montant is aspirant of the Fonds National de la Recherche Scientifique of the Belgian Government. Dr Chenot was supported by the Daman Foundation.

* P.M. and F.C. contributed equally to this work

Received for publication May 19, 2008; revisions received Feb 27, 2009; accepted for publication March 29, 2009; available ahead of print June 29, 2009.

Address for reprints: Jean-Louis Vanoverschelde, MD, PhD, Division of Cardiology, Cliniques Universitaires Saint-Luc, Avenue Hippocrate 10-2881, B-1200 Brussels, Belgium (E-mail: vanoverschelde@card.ucl.ac.be).

$0022-5223 / \$ 36.00$

Copyright (c) 2009 by The American Association for Thoracic Surgery doi:10.1016/j.jtcvs.2009.03.046
}

timing in asymptomatic patients remains controversial. According to current American Heart Association/American College of Cardiology and European Society of Cardiology guidelines,${ }^{3,4}$ surgery is usually not recommended in asymptomatic patients with preserved left ventricular (LV) function. Both sets of guidelines nonetheless indicate that surgery may be considered, but is not compulsory, when atrial fibrillation (AF) ensues or in the presence of pulmonary hypertension.

Despite these recommendations, a number of authors have called for a different standard of referring patients with chronic MR to surgery. ${ }^{5}$ They suggest that if the mitral valve is both severely regurgitant and repairable, postponing surgery exposes the patient to unnecessary risk in an era in which mitral valve repair in high-volume specialized centers has a very low morbidity and mortality. ${ }^{6}$ Thus, they recommend elective surgery early rather than late in such patients. On the other hand, other authors have advocated to postpone surgery until the end points in the guidelines are met. ${ }^{7}$ They argue that, according to most database reports, operative mortality remains relatively high and the majority of patients who have mitral valve surgery ultimately receive a mitral prosthesis, ${ }^{1,8,9}$ which is associated with markedly higher 


$$
\begin{aligned}
& \text { Abbreviations and Acronyms } \\
& \begin{aligned}
\mathrm{AF} & =\text { atrial fibrillation } \\
\mathrm{CABG} & =\text { coronary artery bypass graft surgery } \\
\mathrm{LV} & =\text { left ventricular } \\
\mathrm{MR} & =\text { mitral regurgitation }
\end{aligned}
\end{aligned}
$$

operative mortality and prosthetic valve-related long-term mortality and morbidity. ${ }^{10,11}$ Recent data suggest that such a conservative approach is safe, particularly when patients can be followed closely and diligently in a structured environment by an interested expert. ${ }^{7}$

In view of this controversy, the present study assessed long-term survival, incidence of cardiac complications, factors that predict outcome, and effect of mitral surgery on the long-term prognosis of patients with asymptomatic severe MR amenable to valve repair. In particular, we sought to determine whether an early surgical approach would benefit asymptomatic patients who do not meet any guidelines criteria (ie, patients without LV dilatation or dysfunction, $\mathrm{AF}$, or pulmonary hypertension). In patients initially treated conservatively, we also investigated whether more regular follow-up and stricter application of the guidelines are associated with a better outcome. To test these hypotheses, we conducted a follow-up study of patients with severe MR due to mitral valve prolapse, as delineated by echocardiography.

\section{METHODS \\ Study Population}

The study population consisted of every patient in whom severe ( $\geq$ grade 3$)$ MR was diagnosed with the use of Doppler echocardiography between January 1, 1990, and December 31, 2001, at the Cliniques Universitaires St-Luc in Brussels. Exclusion criteria were age $>85$ years, associated mitral valve stenosis, previous valve surgery, associated congenital heart disease, and significant aortic valve disease. Patients with class I indication for surgery (ie, New York Heart Association class III or IV, LV ejection fraction $<60 \%$, or LV end-systolic internal diameter $>45 \mathrm{~mm}$ ) were also excluded. ${ }^{3,4}$ Patients with coronary artery disease and those who had bypass grafting were not excluded.

Information on postdiagnosis events was obtained for all patients between December 2006 and April 2007. Cardiac events and causes of death were ascertained by contacting the patient's physicians and reviewing death certificates, coroner's reports, or autopsy records.

\section{Echocardiography}

Echocardiographic data were obtained with commercially available ultrasound systems. All patients had a comprehensive examination, including M-mode and 2-dimensional echocardiography, as well as conventional and color Doppler examinations. All tests were conducted by experienced echocardiographers. Mitral valve prolapse was considered in the presence of a Carpentier type II mitral valve dysfunction ${ }^{12}$ (ie, in the presence of excess leaflet motion with part of the leaflet tips overriding the plane of the mitral annulus causing failure of leaflet coaptation). Flail leaflet was diagnosed when the leaflet tip turned outward, becoming concave toward the left atrium, and was considered a definite sign of severe MR.

The severity of MR was assessed semiquantitatively on a scale of $1+$ to $4+$ by an integrated approach that included valve morphology, the size of the regurgitant jet in the left atrium, the proximal regurgitant jet width, and pul- monary venous flow pattern. Severe MR was defined as a holosystolic, grade $\geq 3+$ MR. The approach to semiquantification used in the present study was in agreement with prevailing guidelines at the time of patient's recruitment. $^{13}$

LV diameters, fractional shortening, and ejection fraction, as well as left atrial size and systolic pulmonary artery pressure (using tricuspid regurgitant velocity), were measured as recommended. ${ }^{14}$

\section{Statistical Analysis}

All analyses were conducted using the SPSS software (version 12.0, SPSS Corp, Chicago, Ill). Continuous variables were expressed as mean \pm standard deviation, categorical variables as counts and percentages, and follow-up times as median and range. Differences between groups were analyzed with a one-way analysis of variance. Overall, cardiovascular, and event-free survival functions were computed with the Kaplan-Meier method and compared using the log-rank chi-square test. Cardiac events were defined as cardiac deaths, need for mitral surgery (including reoperations), mitral endocarditis, complete atrioventricular block, nonfatal stroke, and acute coronary syndromes. For each patient included in the study, the corresponding average age- and gender-specific annual mortality of the Belgian general population was obtained. ${ }^{15}$ On the basis of these mortality data, the probability of cumulative expected survival was determined and an expected survival curve was constructed. Several separate subgroup analyses were performed. The first subgroup analysis investigated the effect of frequency of follow-up visits on outcome and appropriateness of operative criteria with prevailing guidelines. A second subgroup analysis aimed at evaluating the prognostic impact of AF, pulmonary hypertension, or both was performed in patients who presented with one or both of these features at the entrance into the study. Finally, a last subgroup analysis was conducted in patients who did not meet any class I or IIa guidelines criteria (ie, patients without LV dilatation or dysfunction, AF, or pulmonary hypertension). ${ }^{3,4}$

All clinical, angiographic, and echocardiographic variables were also proposed for inclusion into a Cox proportional hazards survival model for determination of the factors independently associated with outcome. For this purpose, an interactive stepwise selection procedure using the maximum partial likelihood ratio chi-square statistic $\left(\chi^{2}\right.$ test $)$ to enter $(<.05$ level $)$ or to remove $(>.05$ level) a covariate into the model was used. Variables were entered until no F-to-enter statistics were significant at the $5 \%$ level and until the mean squared error reached a minimum. Differences between groups were analyzed with a one-way analysis of variance.

To overcome the absence of randomization, we performed a propensity analysis. The theoretical basis of propensity score analysis has been published elsewhere. ${ }^{16}$ Briefly, propensity scores are used to create matched pairs or matched sets that are balanced with respect to many observed covariates. The resulting matched sets are heterogeneous within the covariates, but the covariates aim to have uniform distributions in treated and control groups, which makes the groups as a whole comparable. ${ }^{17}$ Propensity score analysis was carried out in our study to estimate the probability that a patient might have early surgery versus conservative treatment and to eliminate the effect of nonrandomization and selection bias. First, logistic regression analysis of several baseline variables was performed to generate a single propensity score for each patient. Practically, we included all possible covariates that we were able to trace and that were expected to be a potential contributing factor for determining whether or not a patient would receive an early surgery strategy or a conservative treatment strategy (Table 1). The model had an area under the receiver operating characteristic curve of 0.79 . Second, we used a subset analysis, in which patients were grouped according to the magnitude of their propensity score into 5 quintiles, after which their characteristics and outcomes were compared within these quintiles (Table 1). Groups with similar propensity scores appeared to be reasonably well matched with respect to all characteristics. We then calculated the 10-year propensity-matched score-adjusted hazards ratio for each end point using a Cox regression model. 
TABLE 1. Number of patients who were matched in each quintile after propensity scoring*

\begin{tabular}{lccc}
\hline \multicolumn{1}{c}{ Quintile } & $\begin{array}{c}\text { Early surgery } \\
(\mathbf{n}=\mathbf{1 2 5})\end{array}$ & $\begin{array}{c}\text { Conservative treatment } \\
(\mathbf{n}=\mathbf{6 7})\end{array}$ & $\boldsymbol{P}$ value \\
\hline 1st quintile (n, \%) & $33(27 \%)$ & $3(4 \%)$ & .11 \\
2nd quintile (n, \%) & $33(26 \%)$ & $6(9 \%)$ & .66 \\
3rd quintile (n, \%) & $25(20 \%)$ & $14(21 \%)$ & .10 \\
4th quintile (n, \%) & $23(18 \%)$ & $15(22 \%)$ & .37 \\
5th quintile (n, \%) & $11(8 \%)$ & $29(43 \%)$ & .11 \\
*Covariates used for individual propensity scores: age, sex, height, weight, smoking, \\
cholesterol value, hypertension, diabetes mellitus, family history of coronary artery \\
disease, creatinine value, atrial fibrillation, left ventricular ejection fraction, heart \\
rate, left atrial size, left ventricular end-diastolic and end-systolic diameters, systolic \\
tricuspid gradient, prolapse type. A $P$ value indicates the statistical difference between \\
the propensity score within each quintile.
\end{tabular}

\section{RESULTS}

\section{Baseline Characteristics}

The clinical characteristics of the 192 patients who met the inclusion criteria are shown in Table 2. Patients were separated into 2 groups according to the initial treatment option. Sixty-seven patients initially treated conservatively were included in the conservative approach group, whereas the remaining 125 patients, who were operated on within the first 3 months after diagnosis, were included in the early surgery group.

In the early surgery group, surgery always consisted of mitral valve repair. Twenty-seven patients also had coronary artery bypass graft surgery (CABG).

\section{Overall and Cardiovascular Survival}

During a median follow-up of 8.5 years, 45 patients died, $31(46 \%)$ in the conservative approach group and $14(11 \%)$ in the early surgery group. Among the 45 patients who died during follow-up, the cause of death was cardiac in 24 patients (postoperative death in 2 , intractable heart failure in 9 , sudden cardiac death in 7 , thromboembolic or hemorrhagic stroke in 3, and miscellaneous factors in 3) and noncardiac in 21 patients. Figure 1 shows the Kaplan-Meier curves for overall survival in the 2 groups of patients, together with that expected in the age- and gender-adjusted Belgian population. Ten-year overall and cardiovascular survival was significantly lower in the conservative approach than in the early surgery group.

Because 27 patients from the early surgery group had combined $\mathrm{CABG}$ and mitral valve repair, we evaluated whether CABG contributed to their better survival. After 10 years of follow-up, patients having combined mitral valve repair and CABG had a poorer overall and cardiovascular survival $(58 \% \pm 12 \%$ and $77 \% \pm 10 \%$, respectively) than patients having mitral valve repair alone $(94 \% \pm 3 \%$ and $97 \% \pm 2 \%$, respectively, both $\log$ rank $<.01$ ).

\section{Need for Surgery and Nonfatal Events}

During follow-up, 23 patients in the conservative approach group had mitral surgery. Mitral surgery was indi-
TABLE 2. Baseline characteristics of the overall population

\begin{tabular}{|c|c|c|c|}
\hline & $\begin{array}{c}\text { Early surgery } \\
(\mathbf{n}=\mathbf{1 2 5})\end{array}$ & $\begin{array}{c}\text { Conservative } \\
\text { approach } \\
(n=67) \\
\end{array}$ & $P$ value \\
\hline Age (y) & $62 \pm 12$ & $64 \pm 15$ & .54 \\
\hline Male gender $(\%)$ & 71 & 69 & .74 \\
\hline Height (m) & $1.72 \pm 0.08$ & $1.70 \pm 0.09$ & .23 \\
\hline Weight (kg) & $72 \pm 12$ & $70 \pm 13$ & .20 \\
\hline Diabetes mellitus (\%) & 7 & 8 & .8 \\
\hline Hypertension (\%) & 33 & 46 & .06 \\
\hline Smoking (\%) & 18 & 27 & .13 \\
\hline Total cholesterol (mg/dL) & $217 \pm 36$ & $213 \pm 43$ & .60 \\
\hline Serum creatinine $(\mathrm{mg} / \mathrm{dL})$ & $0.99 \pm 0.25$ & $0.98 \pm 0.35$ & .71 \\
\hline Prolapse type $(\%)$ & $67 / 12 / 21$ & $70 / 12 / 18$ & .9 \\
\hline LVEDD (mm) & $59 \pm 7$ & $55 \pm 7$ & $<.000$ \\
\hline $\operatorname{LVESD}(\mathrm{mm})$ & $36 \pm 5$ & $33 \pm 5$ & $<.000$ \\
\hline LVEF (\%) & $70 \pm 6$ & $71 \pm 7$ & .21 \\
\hline LA size (mm) & $49 \pm 9$ & $47 \pm 8$ & .06 \\
\hline $\mathrm{AF}$ & $24 \%$ & $18 \%$ & .36 \\
\hline Systolic tricuspid gradient & $33 \pm 13$ & $29 \pm 11$ & .08 \\
\hline
\end{tabular}
$(\mathrm{mm} \mathrm{Hg})$

$\overline{A F \text {, Atrial fibrillation; } L A \text { size, left atrial size; } L V E D D \text {, left ventricular end-diastolic }}$ diameter; $L V E F$, left ventricular ejection fraction; $L V E S D$, left ventricular end-systolic diameter; prolapse type, posterior/anterior/bileaflet.

cated on the basis of class I guidelines criteria in 17 , class IIa guidelines criteria in 2, infective endocarditis in 3 , and need for CABG in 1 . Median delay between the development of guidelines criteria and surgery was 49 days (range 28-139 days). Seven patients in the early surgery group also needed a reoperation, 5 because of recurrent severe MR, 1 because of mitral stenosis, and 1 because of a voluminous atrial thrombus. Eleven additional nonfatal cardiac events (4 strokes, 3 complete atrioventricular blocks, 3 acute myocardial infarctions, and 1 infective endocarditis) also occurred during follow-up. As shown in Figure 1, 10-year cardiac event-free survival was significantly lower in the conservative approach than in the early surgery group.

\section{Impact of AF, Pulmonary Hypertension, or Both on Long-Term Outcome}

A total of 60 patients (16 in the conservative approach group and 44 in the early surgery group) presented with $\mathrm{AF}$, pulmonary hypertension, or both at the time of diagnosis. Their baseline characteristics are shown in Table 3. During follow-up, 20 of these patients died, $13(81 \%)$ in the conservative approach group and $7(16 \%)$ in the early surgery group. The cause of death was cardiac in 13 patients (postoperative death in 2, intractable heart failure in 4 , sudden cardiac death in 3 , thromboembolic or hemorrhagic stroke in 1, and miscellaneous factors in 3) and noncardiac in 7 patients. As shown in Figure 2, 10-year overall, cardiovascular, and cardiac event-free survivals were significantly lower in the conservative approach than in the early surgery group. 

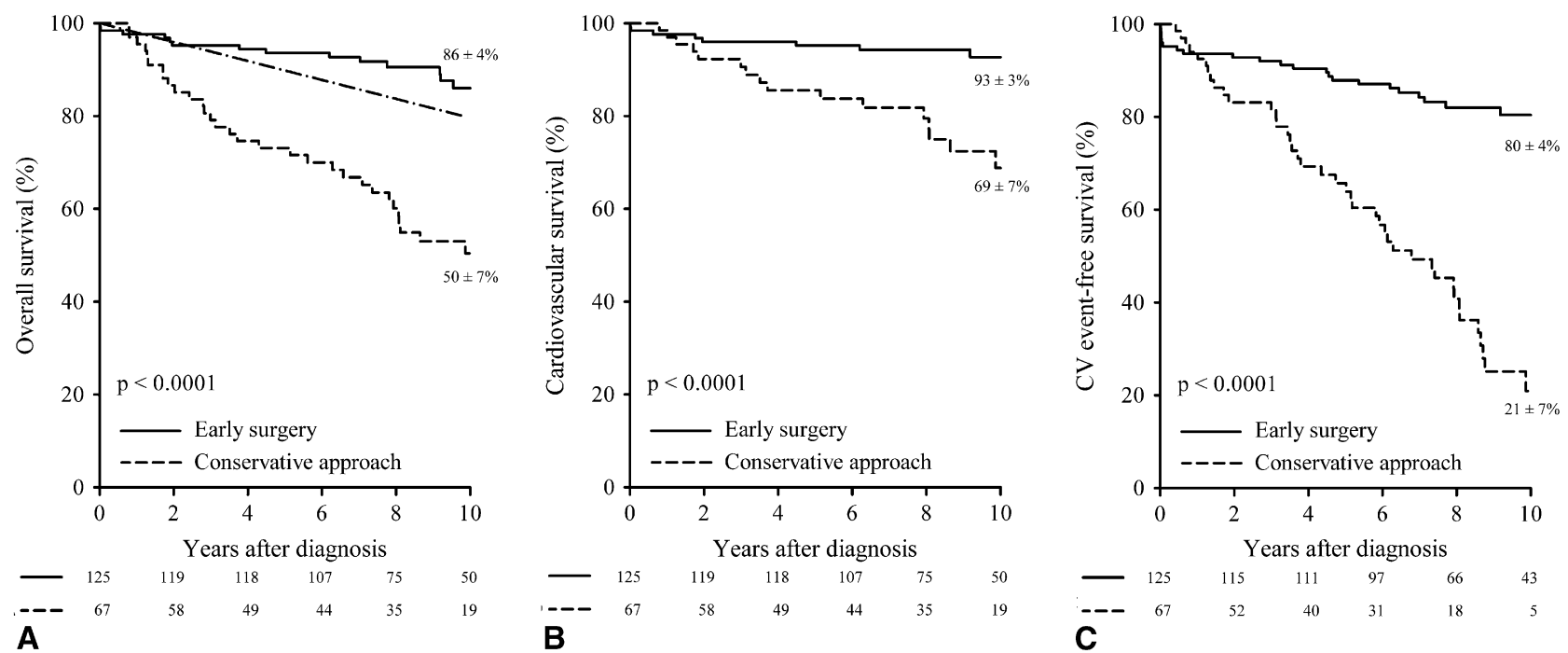

FIGURE 1. Kaplan-Meier curves for overall (A), cardiovascular (B), and cardiovascular (CV) event-free (C) survival compared with patients having early surgery (solid line), those treated conservatively (dashed line), and the age- and gender-matched Belgian population (dashed dotted line). Numbers at bottom indicate patients at risk.

\section{Survival in Patients Free From Any MR Complications}

A total of 132 patients (51 in the conservative approach group and 81 in the early surgery group) were free of any MR complications at the time of diagnosis. Their baseline characteristics are shown in Table 4. During the 10-year follow-up period, 25 of these patients died, $18(35 \%)$ in the conservative approach group and $7(9 \%)$ in the early surgery

TABLE 3. Baseline characteristics of asymptomatic patients with AF, pulmonary hypertension, or both

\begin{tabular}{|c|c|c|c|}
\hline & $\begin{array}{c}\text { Early surgery } \\
(n=44)\end{array}$ & $\begin{array}{c}\text { Conservative } \\
\text { approach } \\
(\mathbf{n}=16) \\
\end{array}$ & $P$ value \\
\hline Age (y) & $67 \pm 11$ & $71 \pm 10$ & .18 \\
\hline Male gender $(\%)$ & 68 & 63 & .76 \\
\hline Height (m) & $1.71 \pm 0.07$ & $1.69 \pm 0.11$ & .38 \\
\hline Weight $(\mathrm{kg})$ & $72 \pm 14$ & $71 \pm 14$ & .87 \\
\hline Diabetes mellitus (\%) & 5 & 6 & .99 \\
\hline Hypertension $(\%)$ & 39 & 19 & .22 \\
\hline Smoking (\%) & 18 & 25 & .72 \\
\hline Total cholesterol (mg/dL) & $214 \pm 40$ & $195 \pm 46$ & .17 \\
\hline Serum creatinine $(\mathrm{mg} / \mathrm{dL})$ & $1.10 \pm 0.26$ & $1.10 \pm 0.44$ & .72 \\
\hline Prolapse type (\%) & $73 / 13 / 14$ & $87 / 13 / 0$ & .30 \\
\hline LVEDD (mm) & $58 \pm 7$ & $52 \pm 5$ & .02 \\
\hline $\operatorname{LVESD}(\mathrm{mm})$ & $36 \pm 5$ & $31 \pm 5$ & $<.0001$ \\
\hline LVEF $(\%)$ & $68 \pm 7$ & $71 \pm 8$ & .20 \\
\hline LA size (mm) & $50 \pm 9$ & $52 \pm 10$ & .59 \\
\hline $\mathrm{AF}(\%)$ & 68 & 75 & .99 \\
\hline $\begin{array}{l}\text { Systolic tricuspid } \\
\text { gradient }(\mathrm{mm} \mathrm{Hg})\end{array}$ & $42 \pm 14$ & $37 \pm 13$ & .25 \\
\hline Pulmonary hypertension (\%) & 38 & 38 & .99 \\
\hline
\end{tabular}

$A F$, Atrial fibrillation; $L A$ size, left atrial size; $L V E D D$, left ventricular end-diastolic diameter; $L V E F$, left ventricular ejection fraction; $L V E S D$, left ventricular end-systolic diameter; prolapse type, posterior/anterior/bileaflet. group. The cause of death was cardiac in 11 patients (intractable heart failure in 5, sudden cardiac death in 4 , and thromboembolic or hemorrhagic stroke in 2) and noncardiac in 14 patients. During follow-up, 20 patients in the conservative approach group had mitral surgery, whereas 6 patients in the early surgery group needed a reoperation. Eight additional nonfatal cardiac events ( 2 strokes, 3 complete atrioventricular blocks, 2 acute myocardial infarctions, and 1 infective endocarditis) also occurred. As shown in Figure 3, 10-year overall, cardiovascular, and cardiac event-free survival was significantly lower in the conservative approach than in the early surgery group.

\section{Effects of Follow-up Regularity and Quality on Outcome in the Conservative Approach Group}

To evaluate the potential influence of the regularity and quality of follow-up visits on outcome, patients from the conservative approach group were further subdivided into 2 subgroups according to whether they were seen by a registered cardiologist at least once a year $(n=43)$ or not $(n=$ 24). Among the 43 patients who were regularly followed, 23 had mitral surgery during follow-up. Indications for surgery were reported above. Among the 20 patients who were regularly followed but were not operated on, 4 also developed class I or IIa indications for surgery but were denied surgery on the basis of age-related comorbidities. Interestingly, none of the patients with irregular follow-up had mitral surgery during follow-up. As shown in Figure 4, patients having a regular follow-up had a significantly better 10 -year overall survival than patients having no follow-up or having irregular follow-up. Despite this, overall 10-year survival was less in regularly followed patients from the conservative approach group than in patients having early surgery. 

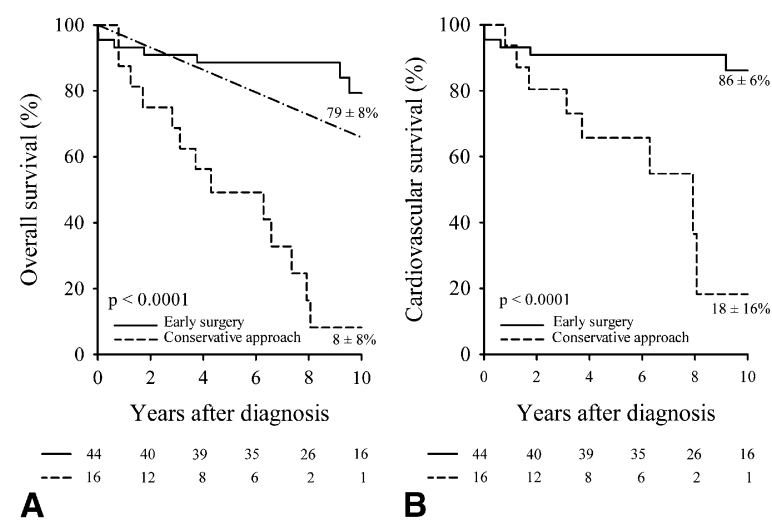

B

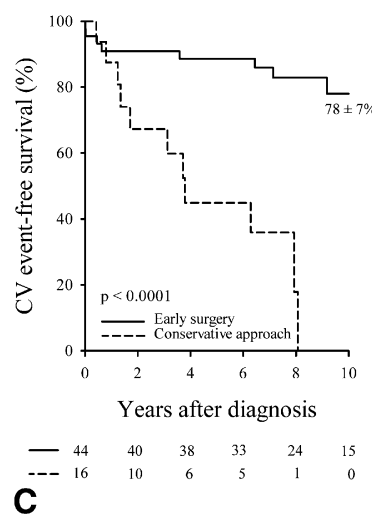

FIGURE 2. Kaplan-Meier curves for overall (A), cardiovascular (B), and cardiovascular $(C V)$ event-free (C) survival compared with patients with atrial fibrillation, pulmonary hypertension, or both who had early surgery (solid line) or were treated conservatively (dashed line). Numbers at bottom indicate patients at risk. The dashed dotted line represents the age- and gender-matched Belgian population.

\section{Cox Proportional-Hazards Survival Analysis}

To assess the factors potentially associated with 10 -year overall and cardiovascular survival, 2 different Cox survival models were built to which all the clinical and echocardiographic parameters available were proposed for inclusion. Among these variables, only the initial treatment strategy and age were independently associated with the time to death (Table 5). Similar results were obtained after removal of the patients who had concomitant CABG $(\mathrm{n}=32)$ from both the early surgery and conservative approach groups (hazards ratio treatment strategy, 9.25 [3.53-24.21]; hazards ratio age, 1.08 [1.04-1.11]). Figure 5 shows the age-adjusted Kaplan-Meier curves for overall survival in the 2 groups of patients, together with that expected in the age- and gender-adjusted Belgian

TABLE 4. Baseline characteristics of asymptomatic patients without LV dysfunction, LV dilatation, AF, or pulmonary hypertension

\begin{tabular}{lccc}
\hline & $\begin{array}{c}\text { Early surgery } \\
(\mathbf{n = 8 1})\end{array}$ & $\begin{array}{c}\text { Conservative } \\
\text { approach } \\
(\mathbf{n}=\mathbf{5 1})\end{array}$ & $\boldsymbol{P}$ value \\
\hline Age (y) & $60 \pm 13$ & $61 \pm 16$ & .57 \\
Male gender (\%) & 73 & 71 & .84 \\
Height (m) & $1.72 \pm 0.09$ & $1.70 \pm 0.09$ & .33 \\
Weight (kg) & $73 \pm 11$ & $70 \pm 12$ & .13 \\
Diabetes mellitus (\%) & 9 & 8 & .99 \\
Hypertension (\%) & 30 & 55 & .003 \\
Smoking (\%) & 19 & 28 & .28 \\
Total cholesterol (mg/dL) & $218 \pm 34$ & $218 \pm 41$ & .95 \\
Serum creatinine (mg/dL) & $0.96 \pm 0.24$ & $0.94 \pm 0.31$ & .74 \\
Prolapse type (\%) & $64 / 11 / 25$ & $65 / 12 / 23$ & .99 \\
LVEDD (mm) & $60 \pm 8$ & $56 \pm 7$ & .002 \\
LVESD (mm) & $36 \pm 5$ & $34 \pm 5$ & .004 \\
LVEF (\%) & $70 \pm 6$ & $71 \pm 7$ & .55 \\
LA size (mm) & $49 \pm 8$ & $45 \pm 7$ & .012 \\
Systolic tricuspid gradient & $28 \pm 8$ & $25 \pm 7$ & .12 \\
$\quad$ (mm Hg) & & &
\end{tabular}

$\overline{A F \text {, Atrial fibrillation; } L A \text { size, left atrial size; } L V E D D \text {, left ventricular end-diastolic }}$ diameter; $L V E F$, left ventricular ejection fraction; $L V E S D$, left ventricular end-systolic diameter; prolapse type, posterior/anterior/bileaflet. population. Even after adjustment for age, the 10-year overall and cardiovascular survivals remained significantly lower in the conservative approach than in the early surgery group.

\section{Propensity Analysis}

The 10-year propensity-matched score adjusted hazards ratio was calculated for each end point using a Cox regression model. The results are shown in Table 6.

\section{DISCUSSION}

The management of asymptomatic patients with severe MR is an area of intense controversy because there are no randomized trials to support any particular course of action. On the one hand, the good results of valve repair and the potential risk of postoperative $\mathrm{LV}$ dysfunction are incentives for early surgery. ${ }^{5,18,19}$ On the other hand, even in low-risk cases, there is a small but definite risk of surgical mortality. ${ }^{8-10}$ To provide new insights into this controversy, we performed a follow-up study of asymptomatic patients with severe degenerative MR amenable to valve repair who were diagnosed at our institution between 1990 and 2001. Our results can be summarized as follows:

1. On average, asymptomatic patients who had early surgical correction of severe degenerative MR incurred lower mortality and morbidity than patients who were followed more conservatively. They also tended to have better overall survival than that expected in the age- and gender-matched Belgian population.

2. Among asymptomatic patients with severe degenerative MR who were not operated on early, those who were regularly followed by a registered cardiologist had a higher likelihood of being referred in a timely manner to surgery and hence had better long-term outcome than those who were not.

3. Asymptomatic patients presenting with AF, pulmonary hypertension, or both had an extremely poor prognosis when treated conservatively. 

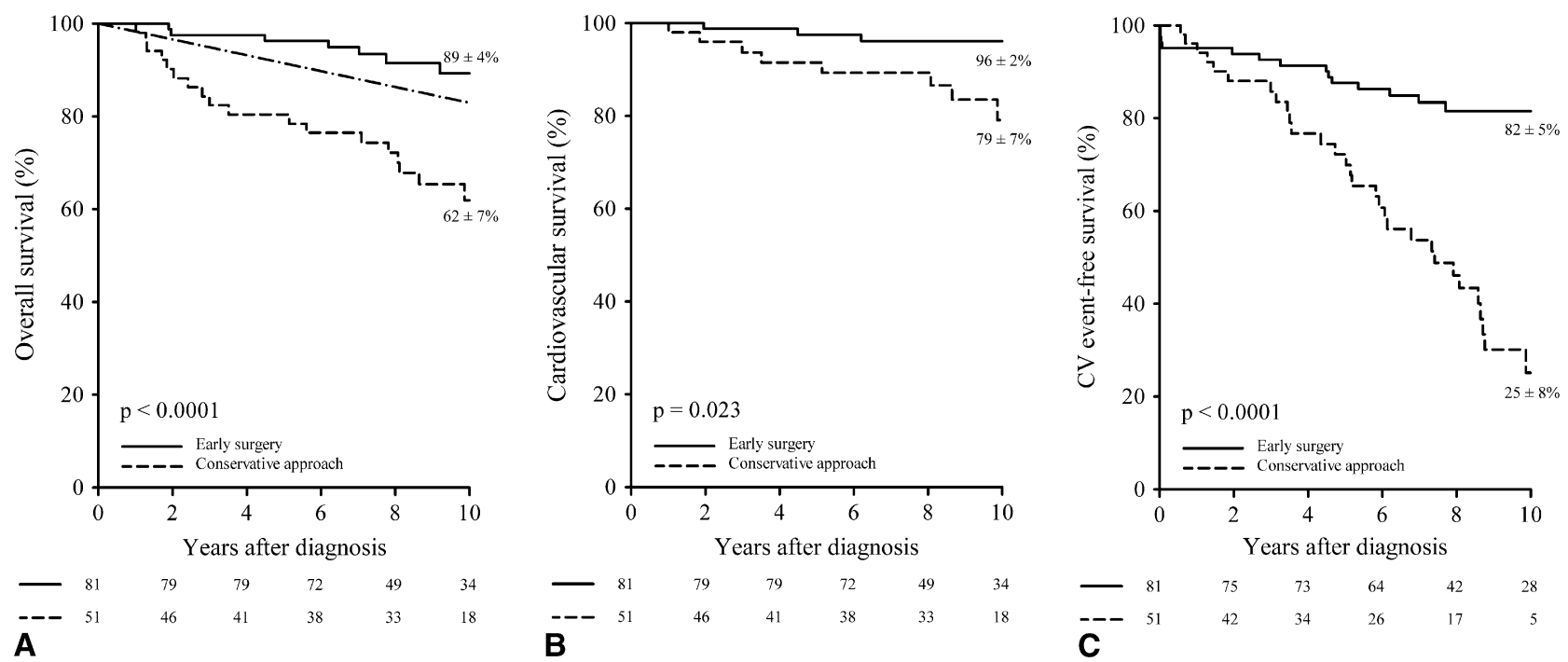

FIGURE 3. Kaplan-Meier curves for overall (A), cardiovascular (B), and cardiovascular $(C V)$ event-free (C) survival compared with patients without left ventricular dysfunction, left ventricular dilatation, atrial fibrillation, or pulmonary hypertension who had early surgery (solid line) and or were treated conservatively (dashed line). Numbers at bottom indicate patients at risk. The dashed dotted line represents the age- and gender-matched Belgian population.

4. In asymptomatic patients with uncomplicated MR (ie, without $\mathrm{LV}$ dilatation or dysfunction, $\mathrm{AF}$, or pulmonary hypertension), early surgery was associated with better overall, cardiovascular, and event-free survival.

\section{Rationale for Early Intervention in Severe Degenerative MR}

To consider surgical intervention early in a disease process, it must be demonstrated that the surgical procedure improves the overall survival and quality of life in comparison to the disease process natural course. Degenerative MR is a progressive disorder ${ }^{20}$ that increases the LV filling pressures, causes enlargement of the left atrium and ventricle, and eventually leads to LV dysfunction and congestive heart failure. ${ }^{19}$ Previous studies have demonstrated that the presence of LV dysfunction or heart failure in a preoperative patient negatively affects the postoperative outcome, ${ }^{21,22}$ suggesting that surgical correction should be performed before the development of the functional impairment.

For such a strategy to be applicable, the immediate and long-term benefits of mitral valve surgery should largely
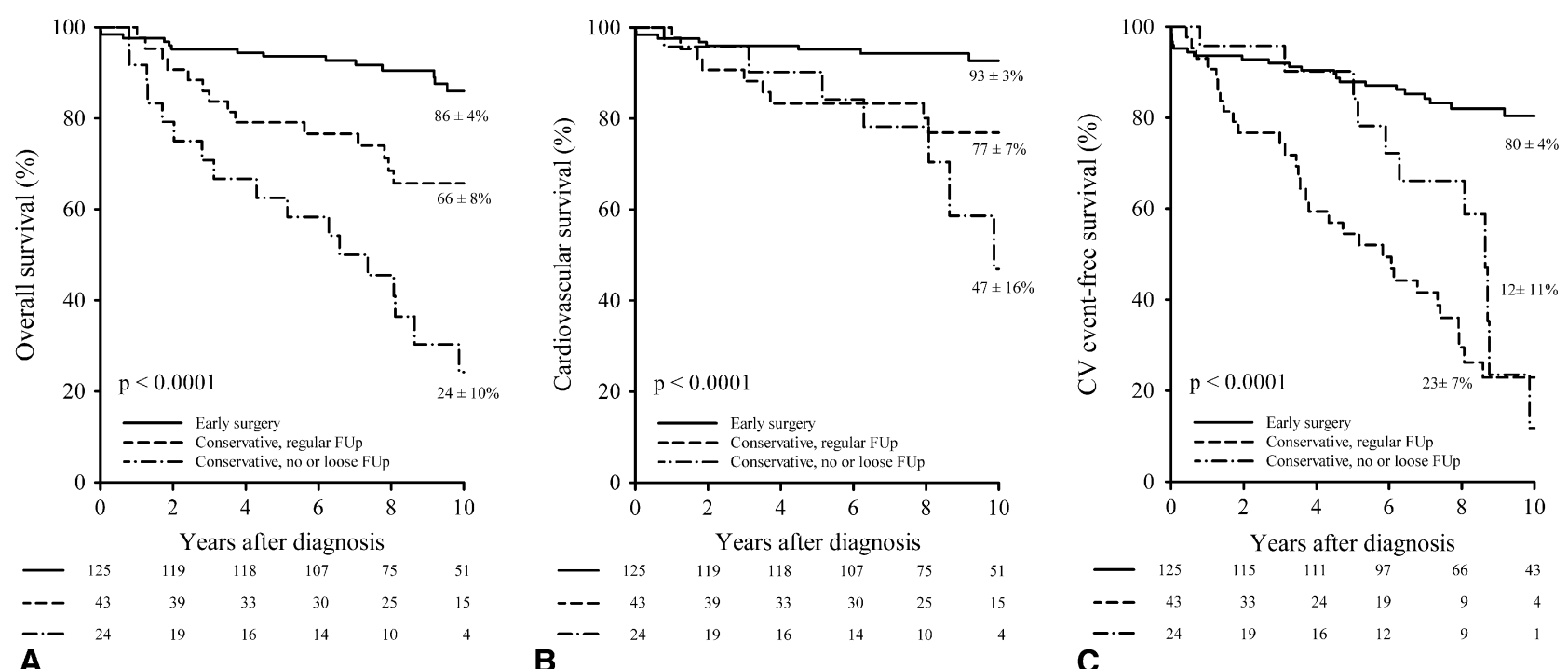

FIGURE 4. Kaplan-Meier curves for overall (A), cardiovascular (B), and cardiovascular (CV) event-free (C) survival compared with patients having early surgery (solid line), those treated conservatively and followed regularly (dashed line), or those treated conservatively and with irregular follow-up (dashed dotted line). Numbers at bottom indicate patients at risk. 
TABLE 5. Cox proportional hazards analysis

\begin{tabular}{|c|c|c|c|c|}
\hline & \multicolumn{2}{|c|}{ Univariate Analysis } & \multicolumn{2}{|c|}{ Multivariate Analysis } \\
\hline & HR $(95 \%$ CI $)$ & $P$ value & HR $(95 \%$ CI $)$ & $P$ value \\
\hline \multicolumn{5}{|l|}{ Overall mortality } \\
\hline Conservative approach & $4.83(2.56-9.00)$ & $<.0001$ & $5.84(2.85-11.9)$ & $<.0001$ \\
\hline Age & $1.11(1.06-1.15)$ & $<.0001$ & $1.07(1.04-1.11)$ & .0001 \\
\hline LVEDD & $0.93(0.89-0.97)$ & .001 & & \\
\hline LVESD & $0.89(0.84-0.95)$ & .001 & & \\
\hline $\mathrm{AF}$ & $2.02(1.09-3.76)$ & .03 & & \\
\hline Hypertension & $0.45(0.22-0.91)$ & .03 & & \\
\hline \multicolumn{5}{|l|}{ Cardiac mortality } \\
\hline Conservative approach & $4.31(1.84-10.10)$ & .001 & $3.37(1.22-9.26)$ & .019 \\
\hline Age & $1.16(1.09-1.23)$ & $<.0001$ & $1.15(1.07-1.24)$ & $<.0001$ \\
\hline Serum creatinine & $4.23(1.81-9.9)$ & .001 & & \\
\hline LVEDD & $0.94(0.89-0.99)$ & .02 & & \\
\hline $\mathrm{AF}$ & $2.89(1.23-6.51)$ & .01 & & \\
\hline
\end{tabular}

outweigh the operative risk as well as the long-term valverelated morbidity and mortality risks. In the past, the operative mortality of mitral valve replacement was undoubtedly too high to consider surgery in asymptomatic patients. ${ }^{12}$ However, with the advent of mitral valve repair, operative mortality has considerably decreased. As early as in 1996, Sousa and colleagues ${ }^{23}$ reported surgical results on a series of asymptomatic or mildly symptomatic patients who had mitral valve repair for severe MR. The overall operative mortality in these patients was $1.7 \%, 5$-year freedom from reoperation was $97 \%$, and 5-year event-free survival was $91 \%$. More recently, Ling and colleagues ${ }^{24}$ Smolens and associates, ${ }^{9}$ and David and coworkers ${ }^{6}$ reported very similar results, with operative mortality ranging from $0 \%$ to $0.5 \%$ and 5 -year overall survival ranging from $90 \%$ to $100 \%$.

The good results of valve repair thus make it conceivable to propose this operation to low-risk, still asymptomatic patients. The results of the present study fully support this new paradigm. Degenerative mitral valve disease in our series was $100 \%$ repairable with the initial operative procedure. There were no patients in our series who had an initial preoperative plan of valve repair that ultimately required valve replacement. In our series, only 2 patients with marked pulmonary hypertension died in the immediate postoperative period (30-day mortality of $1.5 \%$ ). Importantly, there was no death among patients who were free of any MR complications at the time of intervention. Long-term survival was
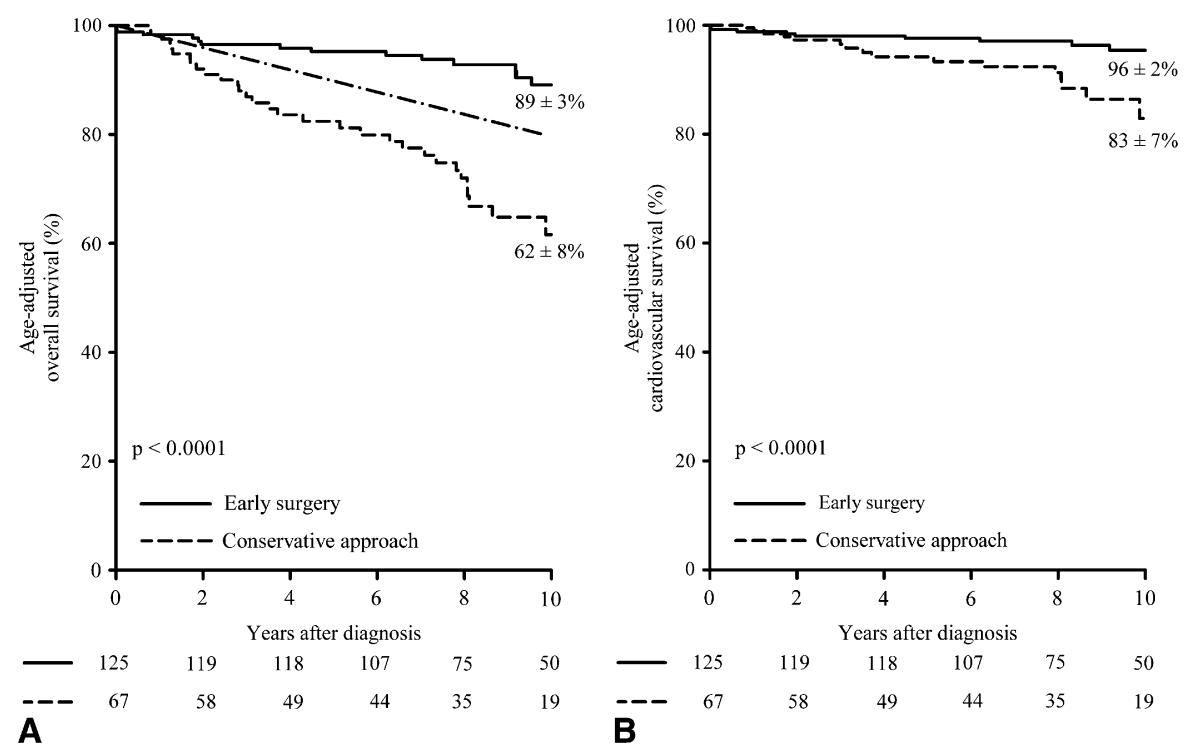

FIGURE 5. Age-adjusted Kaplan-Meier curves for overall (A) and cardiovascular (B) survival compared with patients having early surgery (solid line), those treated conservatively (dashed line), and the age- and gender-matched Belgian population (dashed dotted line). Numbers at bottom indicate patients at risk. 
TABLE 6. Ten-year propensity-matched score-adjusted hazards ratio for overall mortality, cardiac mortality, and cardiovascular events for the conservative treatment

\begin{tabular}{lcc}
\hline \multicolumn{1}{c}{ End point } & 10-year HR & 95\% CI \\
\hline Overall mortality & 5.21 & $2.56-10.60$ \\
Cardiac mortality & 4.83 & $1.84-12.6$ \\
CV events & 4.40 & $2.00-7.90$ \\
\hline
\end{tabular}

$H R$, Hazards ratio; $C I$, confidence interval; $C V$, cardiovascular.

equally good and similar to other reports in the literature. ${ }^{6,9,24}$ Indeed, in our whole series, 5- and 10-year overall survival was $94 \%$ and $86 \%$, respectively. Results were even better in the subgroup of patients who were free of any MR complications. In these patients, 5- and 10-year overall survival was $96 \%$ and $89 \%$, respectively. Based on the results of our study as well as those published in the literature, both the short- and long-term perioperative risks of mitral valve repair appear to be minimal, allowing this procedure to be proposed to low-risk patients with no or minimal symptoms.

\section{Natural History of Severe Degenerative MR}

The natural history of medically treated MR is difficult to delineate, with a wide range of 5-year survival estimates reported in the literature (from $50 \%$ to $90 \%$ ). In a recent study, Enriquez-Sarano and colleagues ${ }^{5}$ studied the outcomes of 198 asymptomatic patients with severe isolated MR who were initially treated medically. MR severity was assessed by the proximal isovelocity surface area method and defined as severe when the regurgitant volume was $>60 \mathrm{~mL}$ and/or the effective regurgitant orifice area was $>40 \mathrm{~mm}^{2}$. In these patients, overall 5-year survival probability was only $58 \%$, and most fatalities were of cardiac origin. The results of the present study confirm those of that earlier report, with an overall 5- and 10-year survival of $60 \%$ and $24 \%$, respectively, in the subgroup of conservatively treated patients who did not have structured follow-up. Similar results were also obtained when censoring conservatively followed patients at the time of the delayed surgery (data not shown). Like in the study of Enriquez-Sarano and colleagues, ${ }^{5}$ the survival rate of patients with severe degenerative MR in our study was significantly less than that of the age- and gender-matched Belgian population, and most fatalities were cardiac related.

The results of these previous studies are clearly at variance with those recently reported by Rosenheck and associates. $^{7}$ These authors followed a cohort of 132 consecutive asymptomatic patients with severe degenerative MR. All patients prospectively had serial clinical and echocardiographic examinations and were referred for surgery when the operative criteria described in the guidelines were fulfilled. During follow-up, 38 patients developed criteria for surgery and only 8 deaths were observed. Overall survival including perioperative and late deaths after mitral valve surgery was $91 \%$ at 8 years and was not statistically differ- ent from expected survival. This suggests that the outcome of patients with severe MR is better than previously reported, particularly when patients are followed closely in a structured environment and are referred in a timely manner to surgery according to current guidelines criteria. The present study, however, questions the good outcome of asymptomatic patients with severe MR even when they are being carefully followed and referred in a timely manner to surgery. To mimic the organized follow-up of Rosenheck and colleagues, ${ }^{7}$ we evaluated whether patients who consulted a registered cardiologist at least once a year had better spontaneous outcomes than patients who did not. Although our results indicate that the frequency and quality of follow-up favorably influences the prognosis of patients treated conservatively, they also show that the overall survival probability of these patients is still significantly less than that of patients operated on early after the index diagnosis. Even after adjustment for all the baseline characteristics using a propensity score analysis, we found that patients having early surgery had a significantly better 10-year overall, cardiac, and cardiovascular event-free survival than those followed conservatively (10-year overall mortality propensity-matched score-adjusted hazards ratio for the conservative group was 5.21 [2.56-10.60]). It could of course be argued that our follow-up was not as strict as that organized by Rosenheck and coworkers, ${ }^{7}$ which could explain why our patient's outcome was poorer. The fact that most of our patients were referred to surgery in a timely manner (within a median of 46 days of newly appearing guidelines criteria) strongly suggests that this explanation is unlikely.

\section{Impact of AF and Pulmonary Hypertension}

Although AF and pulmonary hypertension are common complications of chronic severe MR, their implications for patient management remains uncertain. Indeed, current American Heart Association/American College of Cardiology and European Society of Cardiology guidelines merely consider AF and pulmonary hypertension as a class IIa indication, which indicates that there is no clear consensus among experts to propose surgery in these patients. ${ }^{3,4} \mathrm{Al}-$ though most previous studies have demonstrated that the presence of AF and pulmonary hypertension negatively affects outcome in patients with severe MR, ${ }^{25,26}$ the impact of surgery in these particular patients remains largely unknown. To the best of our knowledge, the present study is the first to address this issue. Our results confirm that the presence of AF, pulmonary hypertension, or both has a negative impact on the survival probability in patients initially treated conservatively. Our results also show that neither of these complications significantly reduces life expectancy in patients who are operated on early after the index diagnosis. Despite the small number of asymptomatic patients presenting with these complications in our study, we feel that on 
the basis of our results, the indication for surgery in the presence of AF and/or pulmonary hypertension should probably be classified as class I instead of the current class IIa.

\section{Survival in Patients Free From Any MR Complications}

The present study is the first to report outcome data in asymptomatic patients with severe MR but without LV dilatation or dysfunction, AF, or pulmonary hypertension. All previous studies indeed included a small but definite number of patients presenting with LV dilatation, LV dysfunction, $\mathrm{AF}$, pulmonary hypertension, or a combination thereof. ${ }^{5-7,9}$ The large number of patients without any of these complications in our study clearly reflects our bias toward early surgical intervention in this patient population. This strategy is supported by the excellent short- and long-term results obtained with early surgery in this group of patients. In our series, there were no perioperative deaths and only 4 patients experienced a perioperative morbid event, for a cumulative operative morbidity risk of $5 \%$. As indicated above, the 5and 10-year overall survival of these patients was $96 \%$ and $89 \%$, respectively. By comparison, the 5- and 10-year overall survival of similar patients being followed more conservatively was significantly less (ie, $78 \%$ and $62 \%$, respectively). As in other subgroups of asymptomatic patients, these data reinforce the hypothesis that most patients with severe MR should be offered an early surgical option whenever their valve is repairable and a skillful surgeon is available for performing the repair.

\section{Limitations to the Study}

There are several limitations to this study that should be acknowledged. First, despite the completeness of our follow-up data and the prospective nature of the database from which the data were retrieved, our study has a retrospective design and the patients were not randomized between the early surgical strategy and the more conservative approach. Although the different groups were relatively well balanced at baseline, we cannot exclude the possibility that unaccounted confounding factors contributed to our results. There is no doubt that a prospective randomized trial would provide a definite demonstration of the superiority of mitral reconstruction in patients with no symptoms or mild symptoms. Unfortunately, it is quite unlikely that such a trial can ever be conducted in today's time, as it would be judged unethical by many surgeons and cardiologists. Second, patients were deemed asymptomatic or mildly symptomatic on the basis of the New York Heart Association functional classification, which has obvious intrinsic limitations. Ideally, patients should have been classified according to the results of an exercise test. Unfortunately, because of the retrospective design of our study, these data were unavailable in a large majority of our patients. Finally, no quantitative measurements of the regurgitant orifice area, the regurgitant volume, or the vena contracta were used in the present study, mainly because these measures were not part of a standard echocardiographic examination at the time the patients were recruited. Yet, the careful, integrated approach to semiquantitative assessment of MR is in full agreement with the prevailing guidelines. ${ }^{27}$ Finally, it would have been interesting to obtain follow-up echocardiographic data in the 2 groups of patients to get more insights into the mechanisms underlying the observed differences in outcome. Unfortunately, the retrospective design of this work did not allow collecting these data.

Our data demonstrate that overall, cardiovascular, and event-free survival of patients with severe degenerative MR is better when opting for an early surgical rather than for a more conservative treatment strategy. Our data also demonstrate that the survival benefits seen in patients having early surgery are present in all patient's subgroups, including patients without any MR complications (ie, without LV dilatation, $\mathrm{LV}$ dysfunction, $\mathrm{AF}$, or pulmonary hypertension).

\section{References}

1. Iung B, Baron G, Butchaet EG, Delahaye F, Gohlke-Bärwolf C, Levan OW, et al. A prospective survey of patients with valvular heart disease in Europe: the Euro Heart Survey on Valvular Heart Disease. Eur Heart J. 2003;24:1231-43.

2. Singh JP, Evans JC, Levy D, Larson MG, Freed LA, Fuller DL, et al. Prevalence and clinical determinants of mitral, tricuspid and aortic regurgitation. Am J Cardiol. 1999;83:897-902.

3. ACC/AHA 2006 Guidelines for the management of patients with valvular heart disease. J Am Coll Cardiol. 2006;48:1-148.

4. Vahanian A, Baumgartner H, Bax JJ, Butchart E, Dion R, Filippatos G, et al. Guidelines for the management of valvular heart disease. Eur Heart J. 2007;28 230-68.

5. Enriquez-Sarano M, Avierinos JF, Messika-Zeitoun D, Detaint D, Capps M, Nkomo V, et al. Quantitative determinants of the outcome of asymptomatic mitral regurgitation. N Engl J Med. 2005;352:875-83.

6. David TE, Ivanov J, Armstrong S, Rakowski H. Late outcomes of mitral valve repair for floppy valves: implications for asymptomatic patients. J Thorac Cardiovasc Surg. 2003;125:1143-52.

7. Rosenheck R, Rader F, Klaar U, Gabriel H, Krejc M, Kalbeck D, et al. Outcome of watchful waiting in asymptomatic severe mitral regurgitation. Circulation. 2006; 113:2238-44

8. Gammie JS, O'Brien SM, Griffith BP, Ferguson TB, Peterson ED. Influence of hospital procedural volume on care process and mortality for patients undergoing elective surgery for mitral regurgitation. Circulation. 2007;115:881-7.

9. Smolens IA, Pagani FD, Deeb GM, Prager RL, Sonnad SS, Bolling SF. Prophylactic mitral reconstruction for mitral regurgitation. Ann Thorac Surg. 2001;72 1210-5

10. Savage EB, Ferguson TB, DiSesa VJ. Use of mitral valve repair: analysis of contemporary United States experience reported to the Society of Thoracic Surgeons National Cardiac Database. Ann Thorac Surg. 2003;75:820-5.

11. Shuhaiber J, Anderson RJ. Meta-analysis of clinical outcomes following surgical mitral valve repair or replacement. Eur J Cardiothorac Surg. 2007;31:267-75.

12. Carpentier A. Cardiac valve surgery-the "French correction". J Thorac Cardiovasc Surg. 1983;86:323-37.

13. Zoghbi WA, Enriquez-Sarano M, Foster E, Grayburn PA, Kraft CD, Levine RA et al. Recommendations for evaluation of the severity of native valvular regurgitation with two-dimensional and Doppler echocardiography. J Am Soc Echocardiogr. 2003;16:777-802.

14. Lang RM, Bierig M, Devereux RB, Flashskampf FA, Foster E, Pellikka PA, et al Recommendations for chamber quantification: a report from the American Society of Echocardiography's guidelines and standards committee and the chamber quantification writing group. J Am Soc Echocardiogr. 2005;18:1440-63.

15. Direction générale statistique et information économique. Population et ménages. Mortalité en 2002. Available at: http://statbel.fgov.be/pub/d2/p203y2002_fr.pdf. Accessed May 5, 2008. 
16. Rosenbaum PR, Rubin DB. The central role of the propensity score in observational studies for causal effects. Biometrika. 1983;70:41-55.

17. Joffe MM, Rosenbaum PR. Invited commentary: propensity scores. Am J Epidemiol. 1999;150:327-33.

18. Enriquez-Sarano M, Schaff HV, Orszulak TA, Tajik AJ, Bailey KR, Frye RL. Valve repair improves the outcome of surgery for mitral regurgitation. A multivariate analysis. Circulation. 1995;91:1022-8.

19. Lee EM, Shapiro LM, Wells FC. Superiority of mitral valve repair in surgery for degenerative mitral regurgitation. Eur Heart J. 1997;18:655-63.

20. Enriquez-Sarano M, Basmadjian AJ, Rossi A, Bailey KR, Seward JB, Tajik AJ. Progression of mitral regurgitation. A prospective Doppler echocardiographic study. J Am Coll Cardiol. 1999;34:1137-44.

21. Enriquez-Sarano M, Tajik AJ, Schaff HV, Orszulak TA, Bailey KR, Frye RL. Echocardiographic prediction of survival after surgical correction of organic mitral regurgitation. Circulation. 1994;90:830-7.

22. Tribouilloy C, Enriquez-Sarano M, Schaff HV, Orzsulak TA, Bailey KR, Tajik AJ, Frye RL. Impact of preoperative symptoms on survival after surgical correction of organic mitral regurgitation. Rationale for optimizing surgical indications. Circulation. 1999;99:400-5.

23. Sousa Uva M, Dreyfus G, Rescigno G, al Aile N, Mascaqni R, La Marra M, et al. Surgical treatment of asymptomatic and mildly symptomatic mitral regurgitation. J Thorac Cardiovasc Surg. 1996;112:1240-8.

24. Ling LH, Enriquez-Sarano M, Seward JB, Tajik AJ, Schaff HV, Bailey KR, Frye RL. Clinical outcome of mitral regurgitation due to flail leaflet. $N$ Engl J Med. 1996;335:1417-23.

25. Grigioni F, Avierinos JF, Ling LH, Scott CG, Bailey KR, Tajik AJ, et al. Atrial fibrillation complicating the course of degenerative mitral regurgitation: determinants and long-term outcome. J Am Coll Cardiol. 2002;40:84-92.

26. Eguchi K, Ohtaki E, Matsumura T, Tanaka K, Tohbaru T, Iguchi N, et al. Pre-operative atrial fibrillation as the key determinant of outcome of mitral valve repair for degenerative mitral regurgitation. Eur Heart J. 2005;26: 1866-72.

27. AHA/ACC 1998 Guidelines for the Management of Patients With Valvular Heart Disease. Circulation. 1998;98:1949-84. 